\title{
Other frailty assessment instruments
}

\author{
Jakub Kenig \\ Department of General, Oncologic, Gastrointestinal Surgery and Transplantology, I Chair of General Surgery, \\ Jagiellonian University Medical College, Krakow, Poland
}

\begin{abstract}
At present there is strong evidence demonstrating that not chronological age, but the presence of frailty before surgery is associated with a significant increase in postoperative morbidity, mortality, along with increased risk of delirium, disability, increased length of hospital stay and resource use. Therefore, preoperative frailty evaluations should become obligatory prior to high-risk surgery of older patients suffering from cancer. Currently, the golden standard is the full Geriatric Assessment. However, it requires time and, first of all, experience. Various simple frailty screening tools have been developed," however, currently there is no single ideal one. Therefore, there is a constant search for the "holy grail" of preoperative geriatric evaluations. The Tilburg Frailty Indicator, the Edmonton Frail Scale, the Cardiovascular Health Study index, the Clinical Frailty Scale, the Study of Osteoporotic Fractures index and Frailty Index are examples of evaluation tools that have some features of screening scores and the full Geriatric Assessment. In the present article they were characterised briefly to familiarize the reader with the advantages and disadvantages of each.
\end{abstract}

Key words: frailty screening, Tilburg Frailty Indicator, Edmonton Frail Scale, Study of Osteoporotic Fractures Index, Frailty Index

As was mentioned in the previous article, the routine format of current preoperative requirements do not provide the information needed for optimal, tailored treatment of older patients with cancer. Therefore, the Geriatric Assessment (GA) was introduced allowing for a preoperative assessment of the patient's condition, the identification of previously unknown health problems, diagnosis of frailty, and assessment of the likelihood of complications and outcome [1]. However, the GA requires experience, it is time-consuming (although the additional 40 minutes required during the preoperative assessment seems to be a low price to pay for the possibility to decrease perioperative morbidity) and not necessary for all patients [2, 3]. Therefore, various screening tools for frailty have been developed: the Vulnerable Elderly Survey 13 [4], Triage Risk Screening Tool [5], Geriatric 8 [6], Groningen Frailty Index [7], abbreviated Comprehensive Geriatric Assessment [8], Rockwood [9], Balducci [10]. Details of their features were presented in the previous article in the Nowotwory Journal of Oncology - Oncogeriatric (part 8). Frailty screening tools can be very beneficial as they can identify patients at risk of frailty and check for adverse outcomes, particularly in situations where there is a lack of experience in the full GA, in acute admitted patients and with low-/moderate-risk surgery [11]. However, only the full GA currently allows for appropriate and full preoperative evaluation and treatment optimisation.

There are also other evaluation instruments that can be used to determine the frailty status of older patients. Some researchers place them between the screening scores and the full GA. This is not entirely true since these instruments have some of the features of both screening scores and the GA. This article aims to systematise current knowledge on the most commonly used instruments. Following the geriatric approach, the tools were divided into: objective (based on direct measurements), subjective (based on medical interviews) and mixed - table I. 
Table I. Other instruments for frailty assessment used in older cancer patients

\begin{tabular}{lll} 
Subjective tools & Objective tools & Mixed tools \\
\hline Tilburg Frailty Indicator & gait speed & Fried Frailty Phenotype criteria \\
CSHA Clinical Frailty Scale & (as a single measure) & Edmonton Frail Scale \\
& & Study of Osteoporotic Fractures Index \\
& & Frailty Index
\end{tabular}

\section{Subjective tools}

The Tilburg Frailty Indicator (TFI) is a tool proposed by Gobbens et al. [12]. Its concept is based on the definition of frailty being a complex, multidimensional, transitional state of increased vulnerability and loss of adaptive capacity/resistant to external stressors (in one or more domains of human functioning: physical, psychological, social, etc.), resulting in an increased risk of adverse outcomes [13].

The TFI consists of two different parts. The first one addresses sociodemographic characteristics |(sex, age, marital status, education level, monthly income and country of origin) and what the potential determinants of frailty can be. The second part evaluates the components of frailty in the form of 15 self reported questions divided into three categories: physical, psychological and social. The physical domain (0-8 points) comprises eight questions related to physical functioning, unexplained weight loss, difficulty in walking, balance, hearing and vision problems, strength in hands, and physical tiredness. The psychological domain (0-4 points) consists of four items related to cognition, depressive symptoms, anxiety, and coping. In turn, the social domain (0-3 points) comprises three questions related to living alone, social relations and social support. The total score may range from 0 to 15; the higher the score, the more severe the frailty. The frailty state is diagnosed when the total score is even or more than five [12]. The tool is simple (it takes less than 15 minutes to complete), does not require face-to-face contact [14], and it was also validated in Polish [15]. A consensus group on frailty in the year 2013 agreed that the TFI is a well-validated model of the frailty concept [16].

The Clinical Frailty Scale (CFS) [17] was introduced in the second clinical examination of the Canadian Study of Health and Aging as a way to summarise the overall level of fitness/frailty of an older adult after being evaluated by an experienced clinician. It is not a questionnaire, but a way to summarise information regarding the health status of an older person. Assessing physicians assign a score from 1 to 7 based on their own clinical judgment. The scale ranges from very fit to severely frail: 1 = very fit, 2 = well, 3 = well with treated comorbidities, 4 = apparently vulnerable, $5=$ mildly frail (some dependence on others for instrumental activities of daily living), 6 = moderately frail (help needed with instrumental and non-instrumental activities of daily living), 7 = severely frail (total dependence on others for activities of daily living, or terminally ill).

In 2020, the CFS was further revised (version 2.0) with minor clarifying edits to the level descriptions and their correspon- ding labels. Most notably, CFS level 2 changed from "well" to "fit", level 4 from "vulnerable" to "living with very mild frailty", and levels 5-8 were restated as "living with..." mild, moderate, severe, and very severe frailty, respectively [18].

The chart also consists of information on scoring frailty in people with dementia. The degree of frailty generally corresponds to the degree of dementia. In mild dementia patients forget the details of a recent event, although still remembering the event itself, repeating the same question/ story and there is usually some degree of social withdrawal. In moderate dementia short-term memory is very impaired, however, personal care is still performed without any support. In severe and very severe dementia, daily activities cannot be performed without help.

\section{Objective tools}

Gait Speed (as a single measure) [19], the time it takes for patients to walk over 4 meters. Gait sped $<0.8 \mathrm{~m} / \mathrm{s}$ is the cut off point for increased risk of adverse health outcomes. Gait speed $<0.2 \mathrm{~m} / \mathrm{s}$ is the cut off point for extreme frailty. A slow gait speed was an independent predictor of post-operative morbidity in older patients undergoing various abdominal operations due to cancer $[20,21]$.

\section{Mixed tools}

Fried Frailty Phenotype criteria developed by Fried et al. [13] is one of the most widely used frailty assessment tools. It uses five relatively easily measured criteria: unintentional weight loss (4.5 kg in the last 12 months), reporting poor energy (using the Depression Scale of the Center for Epidemiologic Studies, weakness (grip strength stratified according to sex and body mass index quartiles), slowness (based on the time taken to walk $4.6 \mathrm{~m}=15$ feet), adjusted for sex and height), low physical activity level (based on the short version of the Minnesota Leisure Time Activity Questionnaire). The score ranges from 0 to 5. Patients are recognised as being frail if they have three or more criteria, pre-frail in the case of one or two criteria and non-frail if none of the criteria are present, respectively. The study in a population of over 10000 older patients has shown that frailty diagnosed on the basis of the above criteria was associated with an unfavourable prognosis; increased risk of death, hospitalisation, disability and falls during the 3- and 7-year follow-up. The risk was correspondingly lower with one or two of the CHS criteria. Despite the wide application of this method, it has significant limitations affecting the possibility of routine use. It includes criteria that require additional measure- 
ments such as grip strength (using a hand-held dynamometer) and is of little use for immobile patients, as well as for people with significant severity of cognitive disorders.

The role in older oncologic surgical patients is still a matter of debate. In our analysis, comparing eight different frailty tools in older cancer patients undergoing high-risk abdominal surgeries, the Fried Frailty Criteria had only moderate accuracy predicting frailty, 30-day morbidity and mortality [11].

The Edmonton Frail Scale (EFS), developed by Rolfson et al. is a performance-based multidimensional frailty assessment tool that is simple (can be completed within $5 \mathrm{~min}$ ) and easy to use by medical personnel without special geriatric training. It is an 11-question questionnaire which analyses nine domains of frailty (cognition, general health status, functional independence, social support, medication use, nutrition, mood, continence, functional performance), with the maximum score of 17 representing the highest level of frailty. Based on the EFS scores, patients can be classified into five categories, ranging from "fit"(0-3), "vulnerable”(4-5), "mildly frail” (6-7), “moderately frail" (8-9) and "severely frail" ( $\geq 10)$ [22]. The EFS correlates very well with geriatricians' clinical impressions [22, 23], captures appropriately every area of frailty, with a high degree of correlation between the EFS scores and Geriatric Assessment as well as other frailty scales [23]. It has also been shown to be able to predict postoperative outcomes when used as a screening tool in the Caucasian and Asian population. In studies by He Y., Dasgupta M., increasing EFS scores were, independent of the age, associated with increased length of hospital stay, postoperative complications, in various abdominal operations $[24,25]$. Moreover, The European Society of Anaesthesiology recommends the use of the EFS in preoperative evaluation of older patients [26].

The Frailty Index (FI) [27] and its modifications are based on the concept that frailty is a consequence of interrelated physical, psychological and social factors. As deficits accumulate, people become increasingly vulnerable to adverse outcomes. The $\mathrm{Fl}$, which is a continuous measure ranging from 0 to a theoretical maximum of 1.0, is calculated as the number of deficits the patient has, divided by the number of deficits considered. The original version of the $\mathrm{Fl}$, developed by Rockwood K. et al. includes 70 items, possible deficits, clinical signs and symptoms. They range from physical disease to psychological and cognitive problems to limitations in the ability to manage daily activities. Other modifications of the FI with a lower number of items have also been developed, including even an 11 variables list [28]. However, there are studies showing that risk evaluation is significant when at least 50 items are considered. The Fl is also strongly correlated with the risk of postoperative morbidity and mortality in a wide range of oncologic procedures and is now recognised as a risk stratification tool $[29,30]$. The Fl, particularly the original version, takes a lot of time and includes functional dependence as a deficit, which may cause confusion between disability and frailty).
The Osteoporotic Fractures index (SOF index) is a short 3-item instrument, an adaptation of Frieds' frailty phenotype score, and is designed to measure pre-frailty and frailty status. As defined by the SOF index, frailty was identified by the presence of two or more of the following three components: weight loss of $\geq 5 \%$ during the preceding year (regardless of any intention to lose weight), an inability to rise from a chair five times without using arms, and an answer of "no" to the question "Do you feel full of energy?". Patients with no impairments were considered to be robust, and those with one disability were considered to be in a pre-frailty status [31]. The SOF index, among others, was used with success to evaluate gastric cancer patients preoperatively [32].

\section{Conclusion}

Not chronological age but rather frailty is recognised as one of the strongest preoperative predictors of postoperative complications in one of the most recently published meta-analysis [33]. Having a clear understanding of postoperative recovery trajectories is essential for conducting appropriate discussions about treatment plans with patients and family [34]. Mclsaac et al. have observed that almost all older patients are willing to participate in a frailty assessment before going for major surgery [35]. Therefore, preoperative frailty evaluations should become obligatory prior to high-risk surgery of older patients suffering from cancer.

The value of the Geriatric Assessment, the current gold standard for frailty, was shown in many studies. However, its applicability in a busy preoperative clinic setting without experience is difficult. As a result, there is a constant search for the holy grail of preoperative assessments. A recently published systematic review and meta-analysis of 70 studies, presenting the accuracy and feasibility of clinically applied frailty instruments before surgery, concluded that specific frailty scales might be better predictors for some adverse outcomes when compared to others. The Clinical Frailty Score was strongly associated with mortality (a 4.9-fold increase in the odds) and discharge to nursing facility (a 6.3-fold increase in the odds). In turn, the Edmonton Frailty Score was a better predictor of complications (a 2.9-fold increase in the odds) and the frailty phenotype was most strongly associated with postoperative delirium (a 3.8-fold increase in the odds) [36]. At present, we do not have a conclusive answer as to which scale should be used preoperatively, apart from the full Geriatric Assessment. Clinicians should consider factors such as accuracy and feasibility when choosing a frailty instrument. The usefulness of most of them is significantly limited due to the quality of their psychometric properties. According to the COSMIN criteria, only the Frailty Index based on the Geriatric Assessment and the Tilburg Frailty Indicator were characterised by significant fair to excellent quality [37] and these are the scales that I recommend to evaluate older cancer patients before high-risk abdominal surgery. The preferred tool of choice is the Geriatric 
Assessment, but in cases where there is a lack of experience, Frailty Index is more suited.

Conflict of interest: none declared

\author{
Jakub Kenig \\ Jagiellonian University Medical College \\ Department of General, Oncologic, Gastrointestinal Surgery \\ and Transplantology \\ I Chair of General Surgery \\ ul. Jakubowskiego 2 \\ 30-688 Kraków, Poland \\ e-mail:jkenig@cm-uj.krakow.pl
}

Received and accepted:12 Oct 2020

\section{References}

1. Kenig J, Szabat K. Oncogeriatrics (part 7.). Geriatric assessment for older patients with cancer. NOWOTWORY J Oncol. 2020; 70(4): 153-157, doi: 10.5603/njo.2020.0031.

2. Bolle S, Smets EMA, Hamaker ME, et al. Medical decision making for older patients during multidisciplinary oncology team meetings. J Geriatr Oncol. 2019; 10(1): 74-83, doi: 10.1016/j.jgo.2018.07.016, indexed in Pubmed: 30213454.

3. Eamer G, Taheri A, Chen SS, et al. Comprehensive geriatric assessment for older people admitted to a surgical service. Cochrane Database Syst Rev. 2018; 1: CD012485, doi: 10.1002/14651858.CD012485.pub2, indexed in Pubmed: 29385235.

4. Saliba S, Elliott $M$, Rubenstein LA, et al. The Vulnerable Elders Survey (VES-13): A Tool for Identifying Vulnerable Elders in the Community. JAGS. 2001; 49: 1691-1699.

5. Meldon SW, Mion LC, Palmer RM, et al. A brief risk-stratification tool to predict repeat emergency department visits and hospitalizations in older patients discharged from the emergency department. Acad Emerg Med. 2003; 10(3): 224-232, doi: 10.1111/j.1553-2712.2003. tb01996.x, indexed in Pubmed: 12615588.

6. Soubeyran P, Bellera C, Goyard J, et al. Validation of the G8 screening tool in geriatric oncology: The ONCODAGE project. NOWOTWORY J Oncol. 2008; 26: abstr 20568.

7. Slaets JPJ.Vulnerability in the elderly: frailty. Med Clin North Am. 2006; 90(4): 593-601, doi: 10.1016/j.mcna.2006.05.008, indexed in Pubmed: 16843764.

8. Overcash JA, Beckstead J, Moody L, et al. The abbreviated comprehensive geriatric assessment (aCGA) for use in the older cancer patient as a prescreen: scoring and interpretation. Crit Rev Oncol Hematol. 2006; 59(3): 205-210, doi: 10.1016/j.critrevonc.2006.04.003, indexed in Pubmed: 16904902.

9. Rockwood K, Stadnyk K, MacKnight C, et al. A brief clinical instrument to classify frailty in elderly people. Lancet. 1999; 353(9148): 205-206, doi: 10.1016/S0140-6736(98)04402-X, indexed in Pubmed: 9923878.

10. Balducci $L$, Beghe $C$. The application of the principles of geriatrics to the management of the older person with cancer. Crit Rev Oncol Hematol. 2000; 35(3): 147-154, doi: 10.1016/s1040-8428(00)00089-5, indexed in Pubmed: 10960797.

11. Kenig J, Szabat K, Mituś J, et al. Usefulness of eight screening tools for predicting frailty and postoperative short- and long-term outcomes among older patients with cancer who qualify for abdominal surgery. Eur J Surg Oncol. 2020; 46(11): 2091-2098, doi: 10.1016/j. ejso.2020.07.040, indexed in Pubmed: 32800399.

12. Gobbens RJJ, van Assen MA, Santiago LM, et al. The Tilburg Frailty Indicator: psychometric properties. J Am Med Dir Assoc. 2010; 11(5): 344-355, doi: 10.1016/j.jamda.2009.11.003, indexed in Pubmed: 20511102.

13. Fried LP, Tangen CM, Walston J, et al. Cardiovascular Health Study Collaborative Research Group. Frailty in older adults: evidence for a phenotype. J Gerontol A Biol Sci Med Sci. 2001; 56(3): M146-M156, doi: 10.1093/gerona/56.3.m146, indexed in Pubmed: 11253156.

14. Gobbens RJJ, van Assen MA, Luijkx KG, et al. The predictive validity of the Tilburg Frailty Indicator: disability, health care utilization, and quality of life in a population at risk. Gerontologist. 2012; 52(5): 619-631, doi: 10.1093/geront/gnr135, indexed in Pubmed: 22217462.
15. Uchmanowicz I, Jankowska-Polańska B, Łoboz-Rudnicka M, et al. Cross-cultural adaptation and reliability testing of the Tilburg Frailty Indicator for optimizing care of Polish patients with frailty syndrome. Clin Interv Aging. 2014; 9: 997-1001, doi: 10.2147/CIA.S64853, indexed in Pubmed: 25028543.

16. Walston JD, Bandeen-Roche K. Frailty: a tale of two concepts. BMC Med. 2015; 13: 185, doi: 10.1186/s12916-015-0420-6, indexed in Pubmed: 26265077.

17. Rockwood K, Song X, MacKnight C, et al. A global clinical measure of fitness and frailty in elderly people. CMAJ. 2005; 173(5): 489-495, doi: 10.1503/cmaj.050051, indexed in Pubmed: 16129869.

18. Rockwood K, Theou O. Using the Clinical Frailty Scale in Allocating Scarce Health Care Resources. Can Geriatr J. 2020; 23(3): 210-215, doi: 10.5770/cgj.23.463, indexed in Pubmed: 32904824.

19. Abellan van Kan G, Rolland Y, Andrieu S, et al. Gait speed at usual pace as a predictor of adverse outcomes in community-dwelling older people an International Academy on Nutrition and Aging (IANA) Task Force. J Nutr Health Aging. 2009; 13(10): 881-889, doi: 10.1007/s12603-0090246-z, indexed in Pubmed: 19924348.

20. Chandoo A, Chi CH, Ji W, et al. Gait speed predicts post-operative medical complications in elderly gastric cancer patients undergoing gastrectomy. ANZ J Surg. 2018; 88(7-8): 723-726, doi: 10.1111/ans.14325, indexed in Pubmed: 29228514.

21. Pamoukdjian F, Canoui-Poitrine F, Longelin-Lombard C, et al. Diagnostic performance of gait speed, G8 and G8 modified indices to screen for vulnerability in older cancer patients: the prospective PF-EC cohort study. Oncotarget. 2017; 8(31): 50393-50402, doi: 10.18632/oncotarget.17361, indexed in Pubmed: 28881570.

22. Rolfson DB, Majumdar SR, Tsuyuki RT, et al. Validity and reliability of the Edmonton Frail Scale. Age Ageing. 2006; 35(5): 526-529, doi: 10.1093/ ageing/afl041, indexed in Pubmed: 16757522.

23. Perna S, Francis MD, Bologna C, et al. Performance of Edmonton Frail Scale on frailty assessment: its association with multi-dimensional geriatric conditions assessed with specific screening tools. BMC Geriatr. 2017; 17(1): 2, doi: 10.1186/s12877-016-0382-3, indexed in Pubmed: 28049443.

24. Dasgupta $M$, Rolfson DB, Stolee $P$, et al. Frailty is associated with postoperative complications in older adults with medical problems. Arch Gerontol Geriatr. 2009; 48(1): 78-83, doi: 10.1016/j.archger.2007.10.007, indexed in Pubmed: 18068828.

25. HeY, Li LW, Hao Y, et al. Assessment of predictive validity and feasibility of Edmonton Frail Scale in identifying postoperative complications among elderly patients: a prospective observational study. Sci Rep. 2020; 10(1): 14682, doi: 10.1038/s41598-020-71140-5, indexed in Pubmed: 32895396.

26. De Hert S, Staender S, Fritsch G, et al. Pre-operative evaluation of adults undergoing elective noncardiac surgery: Updated guideline from the European Society of Anaesthesiology. Eur J Anaesthesiol. 2018; 35(6): 407-465, doi: 10.1097/EJA.0000000000000817, indexed in Pubmed: 29708905.

27. Rockwood K, Song X, MacKnight C, et al. A global clinical measure of fitness and frailty in elderly people. CMAJ. 2005; 173(5): 489-495, doi: 10.1503/cmaj.050051, indexed in Pubmed: 16129869.

28. Velanovich V, Antoine $H$, Swartz $A$, et al. Accumulating deficits model of frailty and postoperative mortality and morbidity: its application to a national database. J Surg Res. 2013; 183(1): 104-110, doi: 10.1016/j. jss.2013.01.021, indexed in Pubmed: 23415494.

29. Mogal H, Vermilion SA, Dodson R, et al. Modified Frailty Index Predicts Morbidity and Mortality After Pancreaticoduodenectomy. Ann Surg Oncol. 2017; 24(6): 1714-1721, doi: 10.1245/s10434-016-5715-0, indexed in Pubmed: 28058551.

30. Garland M, Hsu FC, Shen P, et al. Optimal Modified Frailty Index Cutoff in Older Gastrointestinal Cancer Patients. Am Surg. 2017; 83(8): 860-865, indexed in Pubmed: 28822392.

31. Ensrud KE, Ewing SK, Cawthon PM, et al. Osteoporotic Fractures in Men Research Group. A comparison of frailty indexes for the prediction of falls, disability, fractures, and mortality in older men. J Am Geriatr Soc. 2009; 57(3): 492-498, doi: 10.1111/j.1532-5415.2009.02137.x, indexed in Pubmed: 19245414.

32. Choe YR, Joh JY, Kim YP. Association between frailty and readmission within one year after gastrectomy in older patients with gastric cancer. J Geriatr Oncol. 2017; 8(3): 185-189, doi: 10.1016/j.jgo.2017.02.002, indexed in Pubmed: 28259489.

33. Watt J, Tricco AC, Talbot-Hamon C, et al. Identifying older adults at risk of harm following elective surgery: a systematic review and meta-analysis. BMC Med. 2018; 16(1): 2, doi: 10.1186/s12916-017-0986-2, indexed in Pubmed: 29325567. 
34. Kim S, Brooks AK, Groban L. Preoperative assessment of the older surgical patient: honing in on geriatric syndromes. Clin Interv Aging. 2015; 10: 13-27, doi: 10.2147/CIA.S75285, indexed in Pubmed: 25565783.

35. Mclsaac DI, Taljaard M, Bryson GL, et al. Frailty as a predictor of death or new disability afer surgery: a prospective cohort study. Ann Surg. 2018; 210: 901-908

36. Aucoin SD, Hao M, Sohi R, et al. Accuracy and Feasibility of Clinically Applied Frailty Instruments before Surgery: A Systematic Review and
Meta-analysis. Anesthesiology. 2020; 133(1): 78-95, doi: 10.1097/ ALN.0000000000003257, indexed in Pubmed: 32243326.

37. Sutton JL, Gould RL, Daley S, et al. Psychometric properties of multicomponent tools designed to assess frailty in older adults: A systematic review. BMC Geriatr. 2016; 16: 55, doi: 10.1186/s12877-016-0225-2, indexed in Pubmed: 26927924. 